\title{
Positive solution for the $(p, q)$-Laplacian systems by a new version of sub-super solution method
}

\author{
Mohammed Moussa \\ Laboratoire :EDP, Algébre et Géométrie Spectrale, Department of Mathematics, \\ Faculty of Sciences, Ibn Tofail University, Kenitra, Morocco \\ Abdelqoddous Moussa \\ Mohammed VI Polytechnic University, Ben Guerir, Morocco, and \\ Hatim Mazan \\ Laboratoire : EDP, Algébre et Géométrie Spectrale, Department of Mathematics, \\ Faculty of Sciences, Ibn Tofail University, Kenitra, Morocco
}

\begin{abstract}
Purpose - In this paper, the authors give a new version of the sub-super solution method and prove the existence of positive solution for a $(p, q)$-Laplacian system under weak assumptions than usually made in such systems. In particular, nonlinearities need not be monotone or positive.

Design/methodology/approach - The authors prove that the sub-super solution method can be proved by the Shcauder fixed-point theorem and use the method to prove the existence of a positive solution in elliptic systems, which appear in some problems of population dynamics.

Findings - The results complement and generalize some results already published for similar problems.

Originality/value - The result is completely new and does not appear elsewhere and will be a reference for this line of research.
\end{abstract}

Keywords Nonlinear PDE system, $p$-Laplacian operator, Sub-super solution method

Paper type Research paper

\section{Introduction}

Consider the following $\left(p_{1}, p_{2}\right)$-Laplacian system,

$$
\left\{\begin{array}{llll}
-\Delta_{p_{1}} u_{1} & =\mu_{1} F_{1}\left(x, u_{1}, u_{2}\right) & \text { in } & \Omega \\
-\Delta_{p_{2}} u_{2} & =\mu_{2} F_{2}\left(x, u_{1}, u_{2}\right) & \text { in } & \Omega \\
u_{1}=u_{2} & =0 & \text { on } & \partial \Omega
\end{array}\right.
$$

$\Omega$ is an open bounded domain of $\mathbb{R}^{N}$ with smooth boundary $\partial \Omega$. For $i=1,2$, $\Delta_{p_{i}}=\operatorname{div}\left(\left|\nabla u_{i}\right|^{p_{i}-2} \nabla u_{i}\right)$ is the $p_{i}$-Laplacian operator, $p_{i}>1, \mu_{i}$ is a positive parameter and $F_{i}: \bar{\Omega} \times \mathbb{R} \times \mathbb{R} \rightarrow \mathbb{R}$ is a continuous function.

Many authors have been interested by the problem (1) in different ways [1-4]. The subsuper solution method, given in [5] by using a monotony argument, is the principal tool used to prove the existence of solution of the problem $(1)$ in $[1,3,4]$. Recently, a new version of the

(C) Mohammed Moussa, Abdelqoddous Moussa and Hatim Mazan. Published in Arab Journal of Mathematical Sciences. Published by Emerald Publishing Limited. This article is published under the Creative Commons Attribution (CC BY 4.0) licence. Anyone may reproduce, distribute, translate and create derivative works of this article (for both commercial and non-commercial purposes), subject to full attribution to the original publication and authors. The full terms of this licence may be seen at http:// creativecommons.org/licences/by/4.0/legalcode

The sub-super solution method
Received 10 March 2021 Revised 8 July 2021 Accepted 8 July 2021 
method of the sub-super solution is given to prove the existence of solution for the $(p(x), q(x))$ Laplacian systems by using the Schaefer's fixed-point theorem [6].

Our main contribution in this article is, in first, to give a new version of the sub-super solution method based on Schauder's famous fixed-point theorem and, in second, use the method to prove the existence of a positive solution of problem (1) under the continuity assumptions on functions $F$ and $G$. The functions $F$ and $G$ need not to be nondecreasing as in [1, 4].

Recall that the sub-super solution method is a topological method, which does not require strong regularity assumptions as the variational method.

The paper is organized as follows: in Section 2, we present some preliminary results and our main results. In Section 3, we study some general problems studied previously. We end our paper by studying some concrete examples.

\section{Preliminaries and main results}

We start by the definition of sub-super solution of the problem (1).

Definition 2.1. We say that $\left(\underline{u}_{1}, \bar{u}_{1}\right),\left(\underline{u}_{2}, \bar{u}_{2}\right) \in\left(W^{1, p_{1}}(\Omega) \cap L^{\infty}(\Omega)\right) \times\left(W^{1, p_{2}}(\Omega) \cap L^{\infty}\right.$ $(\Omega))$ is a pair of sub-super solution of the problem (1) if they satisfy

H1. $\underline{u}_{i} \leq \bar{u}_{i}$ a.e in $\Omega$ and $\underline{u}_{i} \leq 0 \leq \bar{u}_{i}$ on $\partial \Omega$ for $i=1,2$.

H2. $-\Delta_{p_{1}} \underline{u}_{1}-F_{1}\left(x, \underline{u}_{1}, v\right) \leq 0 \leq-\Delta_{p_{1}} \bar{u}_{1}-F_{1}\left(x, \bar{u}_{1}, v\right), \forall v \in\left[\underline{u}_{2}, \bar{u}_{2}\right]$,

H3. $-\Delta_{p_{2}} \underline{u}_{2}-F_{2}\left(x, u, \underline{u}_{2}\right) \leq 0 \leq-\Delta_{p_{2}} \bar{u}_{2}-F_{2}\left(x, u, \bar{u}_{2}\right), \forall u \in\left[\underline{u}_{1}, \bar{u}_{1}\right]$.

Inequalities in $\mathrm{H} 1$ and $\mathrm{H} 2$ are in the weak sense. Where, for $u \leq v$ a.e. in $\Omega$, $[u, v]=\{z: u(x) \leq z(x) \leq v(x)$, a.e. $\in \Omega\}$.

Theorem 2.1. For $i=1,2$, assume that $F_{i}$ is continuous in $\bar{\Omega} \times \mathbb{R}^{2}$. Then, if there exists a pair of sub-super solution of (1) in the sense of Definition (2.1), system (1) has a positive weak solution $\left(u_{1}, u_{2}\right) \in\left[\underline{u}_{1}, \bar{u}_{1}\right] \times\left[\underline{u}_{2}, \bar{u}_{2}\right]$.

Proof. Consider, for $i=1,2$, the truncation operators, $T_{i}: L^{p_{i}}(\Omega) \rightarrow L^{p_{i}}(\Omega)$ defined by

$$
T_{i}\left(z_{i}\right)(x)=\left\{\begin{array}{lll}
\underline{u}_{i}(x) & \text { if } & z_{i}(x) \leq \underline{u}_{i}(x) \\
z_{i}(x) & \text { if } & \underline{u}_{i}(x) \leq z_{i}(x) \leq \bar{u}_{i}(x) \\
\bar{u}_{i}(x) & \text { if } & z_{i}(x) \geq \bar{u}_{i}(x)
\end{array}\right.
$$

then $\forall z_{i} \in L^{p_{i}}(\Omega), T_{i}\left(z_{i}\right) \in\left[\underline{u}_{i}, \bar{u}_{i}\right]$ and $\left\|T_{i}\left(z_{i}\right)\right\|_{\infty} \in\left[0,\left\|\bar{u}_{i}\right\|_{\infty}\right]$. By the continuity of $F_{i}$, there exists a positive constant $C_{i}$ such that

$$
\left|F_{i}\left(x, T_{1}\left(z_{1}\right)(x), T_{2}\left(z_{2}\right)(x)\right)\right| \leq C_{i} .
$$

Let $\mathcal{F}_{i}: L^{p_{i}}(\Omega) \times L^{p_{i}}(\Omega) \rightarrow L^{p_{i}}(\Omega)$ be the Nemytskii operator defined by

$$
\mathcal{F}_{i}\left(u_{1}, u_{2}\right)(x)=F_{i}\left(x, T_{1}\left(u_{1}\right)(x), T_{2}\left(u_{2}\right)(x)\right) .
$$

Then, $\mathcal{F}_{i}$ is $L^{p_{i}}(\Omega)$-bounded. By the dominate convergence theorem and the continuity of $F_{\mathrm{i}}$, we conclude the continuity of $\mathcal{F}_{i}$, and we have $\forall\left(u_{1}, u_{2}\right) \in L^{p_{1}}(\Omega) \times L^{p_{2}}(\Omega)$.

$$
\left\|\mathcal{F}_{i}\left(u_{1}, u_{2}\right)\right\|_{\infty} \leq C_{i} .
$$

Now, fix $\left(z_{1}, z_{2}\right) \in L^{p_{1}}(\Omega) \times L^{p_{2}}(\Omega)$, there exists a unique pair $\left(w_{1}, w_{2}\right) \in W_{0}^{1, p_{1}}(\Omega) \times$ $W_{0}^{1, p_{2}}(\Omega)$ solution of the problem,

$$
\left\{\begin{array}{lll}
-\Delta_{p_{1}} w_{1}=\mathcal{F}_{1}\left(T_{1}\left(z_{1}\right), T_{2}\left(z_{2}\right)\right) & \text { in } & \Omega \\
-\Delta_{p_{2}} w_{2}=\mathcal{F}_{2}\left(T_{1}\left(z_{1}\right), T_{2}\left(z_{2}\right)\right) & \text { in } & \Omega \\
w_{1}=w_{2}=0 & \text { on } & \partial \Omega
\end{array}\right.
$$


Therefore, we can define the operator $S: L^{p_{1}}(\Omega) \times L^{p_{2}}(\Omega) \rightarrow L^{p_{1}}(\Omega) \times L^{p_{2}}(\Omega)$ by $S\left(z_{1}, z_{2}\right)=$ $\left(w_{1}, w_{2}\right)$, where $\left(w_{1}, w_{2}\right)$ is the unique solution of problem (3).

$S$ is a compact operator. Indeed, let $\left(z_{1, n}, z_{2, n}\right)$ be a bounded sequence in $L^{p_{1}}(\Omega) \times L^{p_{2}}(\Omega)$ and $\left(w_{1, n}, w_{2, n}\right)=S\left(z_{1, n}, z_{2, n}\right)$, then $\forall \varphi_{i} \in W_{0}^{1, p_{i}}(\Omega)$

$$
\int_{\Omega}\left|\nabla w_{i, n}\right|^{p_{i}-2} \nabla w_{i, n} . \nabla \varphi_{i}=\int_{\Omega} \mathcal{F}_{i}\left(T_{1}\left(z_{1, n}\right), T_{2}\left(z_{2, n}\right)\right) \varphi_{i} .
$$

If the test function $\varphi_{\mathrm{i}}=w_{i, n}$, by the Sobolev embedding theorem, there exists some constants $K_{i}$ such tat

$$
\left\|w_{i, n}\right\|_{W_{0}^{1, p_{i}}(\Omega)}^{p_{i}}=\int_{\Omega}\left|\nabla w_{i, n}\right|^{p_{i}}=\int_{\Omega} \mathcal{F}\left(T_{1}\left(z_{1, n}\right), T_{2}\left(z_{2, n}\right)\right) w_{i, n} \leq C_{i}\left\|w_{i, n}\right\|_{L^{1}(\Omega)} \leq K_{i}\left\|w_{i, n}\right\|_{W_{0}^{1, p_{i}}(\Omega)} .
$$

Then, $\left(w_{1, n}, w_{2, n}\right)$ is bounded in $W_{0}^{1, p_{1}}(\Omega) \times W_{0}^{1, p_{2}}(\Omega)$. By the compact embedding, there exists a convergent sub-sequence of $\left(w_{1, n}, w_{2, n}\right)$ in $L^{p_{1}}(\Omega) \times L^{p_{2}}(\Omega)$. So, $S$ is compact.

From (4), there exists $L_{i}>0$ such that

$$
\left\|w_{i, n}\right\|_{W_{0}^{1, p_{i}}(\Omega)} \leq \bar{K}_{i}=K_{i}^{\frac{1}{p_{i}^{-1}}} \Rightarrow\left\|w_{i, n}\right\|_{L^{p_{i}(\Omega)}} \leq L_{i}
$$

Remark that in (2), (4), and (5), the constants are independent of the choice of $\left(z_{1}, z_{2}\right)$. Then,

$$
S\left(L^{p_{1}}(\Omega) \times L^{p_{2}}(\Omega)\right) \subset B_{L^{p_{1}}(\Omega) \times L^{p_{2}}(\Omega)}(0, \bar{L}) .
$$

for some $\bar{L}>0$. By the Schauder fixed-point theorem, in $B_{L^{p_{1}}(\Omega) \times L^{p_{2}(\Omega)}}(0, \bar{L})$, there exists a unique $\left(u_{1}, u_{2}\right) \in L^{p_{1}}(\Omega) \times L^{p_{2}}(\Omega)$ such that $S\left(u_{1}, u_{2}\right)=\left(u_{1}, u_{2}\right)$.

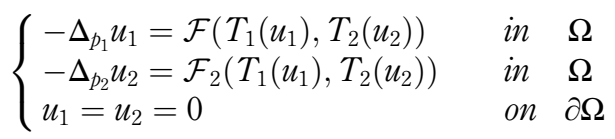

Finally, $\left(u_{1}, u_{2}\right)$ is a solution of problem (1) if, and only if, $T_{1}\left(u_{1}\right)=u_{1}$ and $T_{2}\left(u_{2}\right)=u_{2}$, which means that $\underline{u}_{1} \leq u_{1} \leq \bar{u}_{1}$ and $\underline{u}_{2} \leq u_{2} \leq \bar{u}_{2}$. We need to prove that $\left(\underline{u}_{1}-u_{1}\right)^{+}=0$, $\left(u_{1}-\bar{u}_{1}\right)^{+}=0,\left(\underline{u}_{2}-u_{2}\right)^{+}=0$ and $\left(u_{2}-\bar{u}_{2}\right)^{+}=0$. Let us prove, for example, that $\left(\underline{u}_{1}-u_{1}\right)^{+}=0$. The same argument works for the others cases.

Let $\Omega^{+}=\left\{x \in \Omega, \underline{u}_{1}(x)>u_{1}(x)\right\}$. Since $\left(\underline{u}_{1}, \underline{u}_{2}\right)$ is a sub-solution, then for $\varphi=\left(\underline{u}_{1}-u_{1}\right)^{+}$and $v=T_{2}\left(u_{2}\right)$, we have,

$$
\int_{\Omega}\left|\nabla \underline{u}_{1}\right|^{p_{1}-2} \nabla \underline{u}_{1} \cdot \nabla\left(\underline{u}_{1}-u_{1}\right)^{+} \leq \int_{\Omega} F_{1}\left(x, \underline{u}_{1}, T_{2}\left(u_{2}\right)\right)\left(\underline{u}_{1}-u_{1}\right)^{+}
$$

and as $\left(u_{1}, u_{2}\right)$ is a solution of (6),

$$
\int_{\Omega}\left|\nabla u_{1}\right|^{p_{1}-2} \nabla u_{1} \cdot \nabla\left(\underline{u}_{1}-u_{1}\right)^{+}=\int_{\Omega} F_{1}\left(x, T_{1}\left(u_{1}\right), T_{2}\left(u_{2}\right)\right)\left(\underline{u}_{1}-u_{1}\right)^{+}
$$

Then,

$$
\begin{gathered}
\int_{\Omega}\left(\left|\nabla \underline{u}_{1}\right|^{p_{1}-2} \nabla \underline{u}_{1}-\left|\nabla u_{1}\right|^{p_{1}-2} \nabla u_{1}\right) \cdot \nabla\left(\underline{u}_{1}-u_{1}\right)^{+} \leq \\
\int_{\Omega} F_{1}\left(x, \underline{u}_{1}, T_{2}\left(u_{2}\right)\right)-F_{1}\left(x, T_{1}\left(u_{1}\right), T_{2}\left(u_{2}\right)\right)\left(\underline{u}_{1}-u_{1}\right)^{+} .
\end{gathered}
$$


Remark that in $\Omega^{+}, T_{1}\left(u_{1}\right)=\underline{u}_{1}$. So,

$$
\begin{aligned}
& \int_{\Omega^{+}}\left(\left|\nabla \underline{u}_{1}\right|^{p_{1}-2} \nabla \underline{u}_{1}-\left|\nabla u_{1}\right|^{p_{1}-2} \nabla u_{1}\right) \cdot \nabla\left(\underline{u}_{1}-u_{1}\right)^{+}= \\
& \int_{\Omega^{+}}\left(\left|\nabla \underline{u}_{1}\right|^{p_{1}-2} \nabla \underline{u}_{1}-\left|\nabla u_{1}\right|^{p_{1}-2} \nabla u_{1}\right) \cdot\left(\nabla \underline{u}_{1}-\nabla u_{1}\right) \leq 0 .
\end{aligned}
$$

Therefore, by the monotonicity of the $p_{1}$-Laplacian, $\underline{u}_{1}-u_{1}=0$ in $\Omega^{+}$. Then, $\underline{u}_{1} \leq u_{1}$ in $\Omega$. In the same way, we get $u_{1} \leq \bar{u}_{1}$ in $\Omega$. Then, $\underline{u}_{1} \leq u_{1} \leq \bar{u}_{1}$ and $\underline{u}_{2} \leq u_{2} \leq \bar{u}_{2} \Rightarrow T_{1}\left(u_{1}\right)=u_{1}$ and $T_{2}\left(u_{2}\right)=u_{2}$. Finally, $\left(u_{1}, u_{2}\right)$ is a solution of the problem (1).

\section{Applications}

Theorem 3.1. Consider system (1) and assume that for $i=1,2$,

A.1 $\exists C_{i}, \alpha_{i}, \beta_{i}>0$, such that $\left|F_{i}\left(x, s_{1}, s_{2}\right)\right| \leq C_{i}\left(1+\left|s_{1}\right|^{\alpha_{i}}+\left|s_{2}\right|^{\beta_{i}}\right)$, A. $2 F_{1}(x, 0,0)+F_{2}(x, 0,0)>0$ a.e. $x \in \Omega$.

Then,

(1) If $\max \left(\alpha_{i}, \beta_{i}\right)<p_{i}-1$, for $i=1,2$, then $\forall \mu_{i}>0$, there exists a weak positive solution of problem (1).

(2) If $\min \left(\alpha_{i}, \beta_{i}\right) \geq p_{i}-1$, for $i=1,2$, then, there exists positive numbers $\bar{\mu}_{i}$ such that $\left.\left.\left.\left.\forall\left(\mu_{1}, \mu_{2}\right) \in\right] 0, \bar{\mu}_{1}\right] \times\right] 0, \bar{\mu}_{2}\right]$ the problem (1) has at least a weak positive solution $\left(u_{1}, u_{2}\right)$ such that $\left\|u_{i}\right\|_{\infty} \leq\left\|e_{i}\right\|_{\infty}$. $e_{i}$ is the unique solution of problem (7).

(3) If $\alpha_{i}+\beta_{i}<p_{i}-1$ and $\alpha_{j}+\beta_{j} \geq p_{j}-1 . j=2$ if $i=1$ and $j=1$ if $i=2$. Then there exists $\bar{\mu}_{j}$ such that problem (1) has at least a weak positive solution $\forall \mu_{i}>0$ and $0<\mu_{j} \leq \bar{\mu}_{j}$.

Proof. By A.2, $(0,0)$ is a sub-solution, but not a solution, of problem (1). By Theorem 2.1, we need to find a super solution of problem. Let $e_{i}$ be the unique positive solution of the Dirichlet boundary condition problem,

$$
\left\{\begin{array}{lll}
-\Delta_{p_{i}} u & =1 & \text { in } \Omega \\
u & =0 & \text { on } \partial \Omega
\end{array}\right.
$$

(1) In the sub-linear case, as $\max \left(\alpha_{i}, \beta_{i}\right)<p_{i}-1$, for $i=1$, 2, there exists $K>1$, large enough, such that

$$
\left\{\begin{aligned}
-\Delta_{p_{1}}\left(K e_{1}\right)=K^{p_{1}-1} & \geq \mu_{1} C_{1}\left(1+K^{\alpha_{1}}\left\|e_{1}\right\|_{\infty}^{\alpha_{1}}+K^{\beta_{1}}\left\|e_{2}\right\|_{\infty}^{\beta_{1}}\right) \\
& \geq \mu_{1} C_{1}\left(1+K^{\alpha_{1}}\left\|e_{1}\right\|_{\infty}^{\alpha_{1}}+\|v\|_{\infty}^{\beta_{1}}\right), \\
& \geq \mu_{1} F_{1}\left(x, K e_{1}, v\right), \quad \forall v \in\left[0, K e_{2}\right] \\
-\Delta_{p_{2}}\left(K e_{2}\right)=K^{p_{2}-1} & \geq \mu_{2} C_{2}\left(1+K^{\alpha_{2}}\left\|e_{1}\right\|_{\infty}^{\alpha_{2}}+K^{\beta_{2}}\left\|e_{2}\right\|_{\infty}^{\beta_{2}}\right) \\
& \geq \mu_{2} C_{2}\left(1+\|u\|_{\infty}^{\alpha_{2}}+K^{\beta_{2}}\left\|e_{2}\right\|_{\infty}^{\beta_{2}}\right), \\
& \geq \mu_{2} F_{2}\left(x, u, K e_{2}\right), \quad \forall u \in\left[0, K e_{1}\right]
\end{aligned}\right.
$$


in the weak sense. So, $\left(\bar{u}_{1}, \bar{u}_{2}\right)=\left(K e_{1}, K e_{2}\right)$ is a super-solution of the problem (1). Therefore, there exists $\left(u_{1}, u_{2}\right) \in\left[0, K e_{1}\right] \times\left[0, K e_{2}\right]$ solution of system (1). (2) In the super-linear case, for $i=1,2, \min \left(\alpha_{i}, \beta_{i}\right) \geq p_{i}-1$. Put $\bar{\mu}_{i}=\frac{1}{C_{i}\left(1+\left\|e_{1}\right\|_{\infty}^{\alpha_{i}}+\left\|e_{2}\right\|_{\infty}^{\beta_{i}}\right)}$ and
let $0<\mu_{i} \leq \bar{\mu}_{i}$. Then,

$$
\left\{\begin{aligned}
-\Delta_{p_{1}} e_{1}=1 & =\bar{\mu}_{1} C_{1}\left(1+\left\|e_{1}\right\|_{\infty}^{\alpha_{1}}+\left\|e_{2}\right\|_{\infty}^{\beta_{1}}\right) \\
& \geq \mu_{1} C_{1}\left(1+\left\|e_{1}\right\|_{\infty}^{\alpha_{1}}+\left\|e_{2}\right\|_{\infty}^{\beta_{1}}\right) \\
& \geq \mu_{1} C_{1}\left(1+\left\|e_{1}\right\|_{\infty}^{\alpha_{1}}+v^{\beta_{1}}\right) \\
& \geq \mu_{1} F_{1}\left(x, e_{1}, v\right), \forall v \in\left[0, e_{2}\right] \\
-\Delta_{p_{2}} e_{2}=1 & =\bar{\mu}_{2} C_{2}\left(1+\left\|e_{1}\right\|_{\infty}^{\alpha_{2}}+\left\|e_{2}\right\|_{\infty}^{\beta_{2}}\right) \\
& \geq \mu_{2} C_{2}\left(1+\left\|e_{1}\right\|_{\infty}^{\alpha_{2}}+\left\|e_{2}\right\|_{\infty}^{\beta_{2}}\right) \\
& \geq \mu_{2} C_{2}\left(1+u^{\alpha_{2}}+\left\|e_{2}\right\|_{\infty}^{\beta_{2}}\right) \\
& \geq \mu_{2} F_{2}\left(x, u, e_{2}\right), \forall u \in\left[0, e_{1}\right] .
\end{aligned}\right.
$$

$\left(e_{1}, e_{2}\right)$ is a super-solution, and we deduce that the problem (1) admits a weak positive solution $\left.\left.\left.\left.\left(u_{1}, u_{2}\right), \forall\left(\mu_{1}, \mu_{2}\right) \in\right] 0, \bar{\mu}_{1}\right] \times\right] 0, \bar{\mu}_{2}\right]$ such that $0<\left\|u_{i}\right\|_{\infty} \leq\left\|e_{i}\right\|_{\infty}$.

(3) Consider the sub-super linear case, $0<\alpha_{1}+\beta_{1}<p_{1}-1$ and $\alpha_{2}+\beta_{2} \geq p_{2}-1$ and put $\bar{\mu}_{2}=\frac{1}{C_{2}\left(1+\left\|e_{1}\right\|_{\infty}^{\alpha_{2}}+\left\|e_{2}\right\|_{\infty}^{\beta_{2}}\right)}$. Let $\mu_{1}>0$ and $0<\mu_{2} \leq \bar{\mu}_{2}$. Then, there exists $K$, large enough, such that

$$
\left\{\begin{array}{lll}
-\Delta_{p_{1}}\left(K e_{1}\right) & =K^{p_{1}-1} & \geq \mu_{1} F_{1}\left(x, K e_{1}, v\right), \quad \forall v \in\left[0, K e_{2}\right] \\
-\Delta_{p_{2}} e_{2} & =1 & \geq \mu_{2} F_{2}\left(x, u, e_{2}\right), \quad \forall u \in\left[0, e_{1}\right] .
\end{array}\right.
$$

So, $\left(K e_{1}, e_{2}\right)$ is as super solution of problem $(P)$.

By the same argument, if $\alpha_{1}+\beta_{1} \geq p_{1}-1$ and $0<\alpha_{2}+\beta_{2}<p_{2}-1$. Put $\bar{\mu}_{1}=\frac{1}{C_{1}\left(1+\left\|e_{1}\right\|_{\infty}^{\alpha_{1}}+\left\|e_{2}\right\|_{\infty}^{\beta_{1}}\right)}$. For all $0<\mu_{1} \leq \bar{\mu}_{1}$ and $\mu_{2}>0$, there exists $K$, large enough, such that

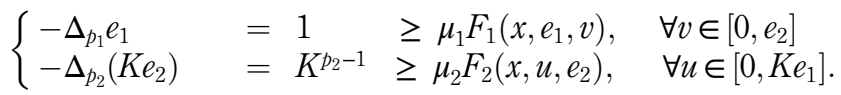

So, $\left(e_{1}, K e_{2}\right)$ is as super solution; hence, the problem (1) admits a weak positive solution $\left(u_{1}, u_{2}\right)$. The proof of Theorem 3.1 is complete.

Remark 3.1. The second point of Theorem 3.1 is of great importance because no restriction was made on the growth of nonlinearities but only on the parameter $\mu_{i}$ which must not be large.

The hypothesis A.2 plays an essential role in the way that we need only to find a super-solution. In what follows, we provide an example without the hypothesis A.2 of Theorem (3.1). We will see that the assumptions on nonlinearities become more restrictive.

Proposition 3.1. For $i=1$, 2, assume that $\exists 0<c_{i} \leq C_{i}, 0<\alpha_{i}, \beta_{i}<p_{i}-1$, $\forall\left(s_{1}, s_{2}\right) \in \mathbb{R}^{+} \times \mathbb{R}^{+}$, 


$$
c_{i} s_{i}^{\alpha_{i}} \leq F_{i}\left(x, s_{1}, s_{2}\right) \leq C_{i}\left(s_{1}^{\alpha_{i}}+s_{2}^{\beta_{1}}\right) .
$$

Then, $\forall \mu_{i}>0$, there exists a weak positive solution of the problem (1).

Proof. According to the proof of Theorem 3.1, there exists $K$, large enough, such that $\left(K e_{1}\right.$, $\left.\mathrm{Ke}_{2}\right)$ is a super-solution of the problem (1). Then, we need only to find a sub-solution. Let $\underline{u}_{i}=\varepsilon \varphi_{i}, \varphi_{i}$ is the principal eigenfunction (positive) associated with the principal eigenvalue of $p_{i}$-Laplacian operator such that $\left\|\varphi_{i}\right\|_{\infty}=1$,

$$
\left\{\begin{array}{lll}
-\Delta_{p_{i}} \varphi_{i} & =\lambda_{1, p_{i}} \varphi_{i}^{p_{i}-1} & \text { in } \Omega \\
\varphi_{i} & =0 & \text { on } \partial \Omega
\end{array}\right.
$$

$\left(\underline{u}_{1}, \underline{u}_{2}\right)$ is as sub-solution of the problem (1). We need to have

$$
\left\{\begin{array}{lll}
-\Delta_{p_{1}} \underline{u}_{1}=\lambda_{1, p_{1}} \varepsilon^{p_{1}-1} \varphi_{1, p_{1}}^{p_{1}-1} & \leq \mu_{1} c_{1} \varepsilon^{\alpha_{1}} \varphi_{1, p_{1}}^{\alpha_{1}} \leq \mu_{1} F_{1}\left(x, \underline{u}_{1}, v\right), & \forall v \in\left[\underline{u}_{2}, \bar{u}_{2}\right] \\
-\Delta_{p_{2}} \underline{u}_{2}=\lambda_{1, p_{2}} \varepsilon^{p_{2}-1} \varphi_{1, p_{2}}^{p_{2}-1} & \leq \mu_{2} c_{2} \varepsilon^{\beta_{2}} \varphi_{1, p_{2}}^{\beta_{2}} \leq \mu_{2} F_{2}\left(x, u, \underline{u}_{2}\right), & \forall u \in\left[\underline{u}_{1}, \bar{u}_{1}\right]
\end{array}\right.
$$

As $\left\|\varphi_{i}\right\|_{\infty}=1$, it is enough to have $\lambda_{1, p_{i}} \varepsilon^{p_{i}-1} \leq \mu_{i} c_{i} \varepsilon^{\delta_{i}}\left(\delta_{1}=\alpha_{1}\right.$ and $\left.\delta_{2}=\beta_{2}\right)$. This is possible for $\varepsilon$, small enough, $\varepsilon<1$ and for all $\mu_{i}>0$. To end the proof, we need to verify that $\varepsilon \varphi_{i}=\underline{u}_{i} \leq \bar{u}_{i}=K e_{i}$ for a small $\varepsilon$ and $K$ large. By the maximum principle, we have

$$
-\Delta_{p_{i}}\left(\varepsilon \varphi_{i}\right)=\varepsilon^{p_{i}-1} \lambda_{1, p_{i}} \varphi_{i} \leq \varepsilon^{p_{i}-1} \lambda_{1, p_{i}} \leq K^{p_{i}-1}=-\Delta_{p_{i}}\left(K e_{i}\right) \Rightarrow \varepsilon \varphi_{i} \leq K e_{i} .
$$

\section{Examples}

In this section, we use Theorem 3.1 and solve some elliptic $\left(\phi_{1}, p_{2}\right)$-Laplacian systems studied in some published articles see [7].

Consider the following $\left(p_{1}, p_{2}\right)$-Laplacian system

$$
(P)\left\{\begin{array}{llll}
-\Delta_{p_{1}} u_{1} & =\mu_{1}\left(a_{1}(x)+b_{1}(x) u_{1}^{\alpha_{1}} u_{2}^{\beta_{1}}\right) & \text { in } & \Omega \\
-\Delta_{p_{2}} u_{2} & =\mu_{2}\left(a_{2}(x)+b_{2}(x) u_{1}^{\alpha_{2}} u_{2}^{\beta_{2}}\right) & \text { in } & \Omega \\
u_{1} & =u_{2}=0 & \text { on } & \partial \Omega
\end{array}\right.
$$

\subsection{The case where $(0,0)$ is a sub-solution}

Assume, for $i=1,2$, that $a_{i}(x)$ and $b_{i}(x)$ are continuous and nonnegative in $\bar{\Omega}, a_{1}$ or $a_{2}$ not identically null. Then, $(0,0)$ is a sub-solution of problem $(P)$. Taking into account Theorem 3.1 and its proof, we get the following propositions,

Proposition 4.1. Problem (P) has a positive weak solution provided that for $i=1,2$,

(1) $\mu_{i}>0$ if $0<\alpha_{i}+\beta_{i}<p_{i}-1$ (The sub-linear case),

(2) $0<\mu_{i} \leq \bar{\mu}_{i}=\frac{1}{C_{i}\left(1+\left\|e_{1}\right\|_{\infty}^{\alpha_{i}}\left\|e_{2}\right\|_{\infty}^{\beta_{i}}\right)}$ if $\alpha_{i}+\beta_{i} \geq p_{i}-1 . C_{i}=\max \left(\left\|a_{i}\right\|_{\infty},\left\|b_{i}\right\|_{\infty}\right)$ (The super linear case) and

(3) $\mu_{i}>0$ and $0<\mu_{j} \leq \bar{\mu}_{j}$ if $\alpha_{i}+\beta_{i}<p_{i}-1$ and $\alpha_{j}+\beta_{j} \geq p_{j}-1 . j=2$ if $i=1$ and $j=1$ if $i=2$ (The sub-super linear case). 
Proof. We have to look for a super solution of our problem.

(1) Since $0<\alpha_{i}+\beta_{i}<p_{i}-1$, for $\forall \mu_{i}>0$, there exists $K$ such that for $i=1,2$, $K^{p_{i}-1} \geq \mu_{i} C_{i}\left(1+K^{\alpha_{i}+\beta_{i}}\left\|e_{1}\right\|_{\infty}^{\alpha_{i}}\left\|e_{2}\right\|_{\infty}^{\beta_{i}}\right)$. Then, we have

$$
\left\{\begin{aligned}
-\Delta_{p_{1}}\left(K e_{1}\right) & =K^{p_{1}-1} \\
& \geq \mu_{1} C_{1}\left[1+\left(K\left\|e_{1}\right\|_{\infty}\right)^{\alpha_{1}}\left(K\left\|e_{2}\right\|_{\infty}\right)^{\beta_{1}}\right] \\
& \geq \mu_{1}\left[a_{1}(x)+b_{1}(x)\left(K e_{1}\right)^{\alpha_{1}}\left(K e_{2}\right)^{\beta_{1}}\right] \\
& \geq \mu_{1}\left[a_{1}(x)+b_{1}(x)\left(K e_{1}\right)^{\alpha_{1}} v^{\beta_{1}}\right], \quad \forall v \in\left[0, K e_{2}\right] .
\end{aligned}\right.
$$

In the same way, we show that $-\Delta_{p_{2}}\left(K e_{2}\right) \geq \mu_{2}\left[a_{2}(x)+b_{2}(x) u^{\alpha_{2}}\left(K e_{2}\right)^{\beta_{2}}\right], \forall u \in\left[0, K e_{1}\right]$. So, $\left(K e_{1}, K e_{2}\right)$ is a super solution of problem $(P)$, and then the problem has a weak positive solution.

(2) Consider the super linear case, for $i=1,2, \alpha_{i}+\beta_{i} \geq p_{i}-1$. Let $0<\mu_{i} \leq \bar{\mu}_{i},\left(e_{1}, e_{2}\right)$ is a super solution of problem $(P)$. Indeed, we have

$$
\left\{\begin{aligned}
-\Delta_{p_{1}} e_{1}=1 & =\bar{\mu}_{1} C_{1}\left[1+\left\|e_{1}\right\|_{\infty}^{\alpha_{1}}\left\|e_{2}\right\|_{\infty}^{\beta_{1}}\right] \\
& \geq \mu_{1} C_{1}\left[1+\left\|e_{1}\right\|_{\infty}^{\alpha_{1}}\left\|e_{2}\right\|_{\infty}^{\beta_{1}}\right] \\
& \geq \mu_{1}\left[a_{1}(x)+b_{1}(x) e_{1}^{\alpha_{1}} e_{2}^{\beta_{1}}\right] \\
& \geq \mu_{1}\left[a_{1}(x)+b_{1}(x) e_{1}^{\alpha_{1}} v^{\beta_{1}}\right], \quad \forall v \in\left[0, e_{2}\right] .
\end{aligned}\right.
$$

In the same way, we obtain $-\Delta_{p_{2}} e_{2} \geq \mu_{2}\left[a_{2}(x)+b_{2}(x) u^{\alpha_{2}} e_{2}^{\beta_{2}}\right], \forall u \in\left[0, e_{1}\right]$. We conclude that problem $(P)$ has a positive weak solution in $\left[0, e_{1}\right] \times\left[0, e_{2}\right]$.

(3) Finally, the third point, the sub-super linear case, follows from the two previous ones.

\subsection{The case where $(0,0)$ is a trivial solution}

We examine only the case where $p_{1}=p_{2}=p$. $(0,0)$ is a trivial solution of $(P)$ if $a_{i}, i=1,2$, are identically null. Our goal is to find a positive solution of problem $(P)$. To do this, we need to find a pair of positive sub-super solution. Nevertheless, we have to add some more assumptions. Assume that, for $i=1,2, b_{\mathrm{i}}$ is positive continuous in $\bar{\Omega}$. So, $c_{i} \leq\left\|b_{i}\right\|_{\infty} \leq C_{i}$ for some positive constants $c_{i}$ and $C_{i}$. The problem becomes

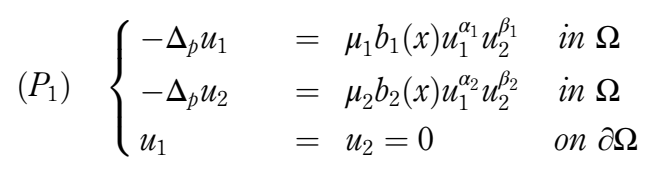

Proposition 4.2. Assume that, for $i=1,2,0<\alpha_{i}+\beta_{i}<p-1$. Then, the problem $\left(P_{1}\right)$ has a positive weak solution $\forall \mu_{i}>0$. 
Proof. According to Theorem 2.1, we need to find a pair of sub-super solution of the problem $\left(P_{1}\right)$. Assume that for $i=1,2,0<\alpha_{i}+\beta_{i}<p-1$, then $\forall \mu_{i}>0$, we can choose $0<\varepsilon<1$, such that $\lambda_{1, p} \varepsilon^{p-1-\left(\alpha_{i}+\beta_{i}\right)} \leq \mu_{i} c_{i}$ for $i=1,2$. Fix such $\varepsilon$ and choose $K \geq \max \left(\lambda_{1, p}^{\frac{1}{p-1}} \varepsilon, 1\right)$ such that $K^{p-1-\left(\alpha_{i}+\beta_{i}\right)} \geq \mu_{i} C_{i}\left\|e_{1}\right\|_{\infty}^{\alpha_{i}+\beta_{i}}$ for $i=1$, 2. Then, $\left(\varepsilon \varphi_{1}, \varepsilon \varphi_{1}\right)$ and $\left(K e_{1}, K e_{1}\right)$ is a pair of sub-super linear solution of the problem $\left(P_{1}\right)\left(\left\|\varphi_{1}\right\|_{\infty}=1\right)$. Indeed, we have, by the maximum principle, $\varepsilon \varphi_{1} \leq K e_{1}$ because

$$
-\Delta_{p}\left(\varepsilon \varphi_{1}\right)=\lambda_{1, p} \varepsilon^{p-1} \varphi_{1}^{p-1} \leq \lambda_{1, p} \varepsilon^{p-1} \leq K^{p-1}=-\Delta_{p}\left(K e_{1}\right) .
$$

$\forall(u, v) \in\left[\varepsilon \varphi_{1}, K e_{1}\right] \times\left[\varepsilon \varphi_{1}, K e_{1}\right]$, we have

$$
\left\{\begin{aligned}
-\Delta_{p}\left(\varepsilon \varphi_{1}\right)=\lambda_{1, p} \varepsilon^{p-1} \varphi_{1}^{p-1} & \leq \mu_{1} c_{1} \varepsilon^{\alpha_{1}+\beta_{1}} \varphi_{1}^{\alpha_{1}+\beta_{1}} \\
& \leq \mu_{1} c_{1}\left(\varepsilon \varphi_{1}\right)^{\alpha_{1}}\left(\varepsilon \varphi_{1}\right)^{\beta_{1}} \\
& \leq \mu_{1} b_{1}(x)\left(\varepsilon \varphi_{1}\right)^{\alpha_{1}} v^{\beta_{1}} . \\
-\Delta_{p}\left(\varepsilon \varphi_{1}\right)=\lambda_{1, p} \varepsilon^{p-1} \varphi_{1}^{p-1} & \leq \mu_{2} c_{2} \varepsilon^{\alpha_{2}+\beta_{2}} \varphi_{1}^{\alpha_{2}+\beta_{2}} \\
& \leq \mu_{2} c_{2}\left(\varepsilon \varphi_{1}\right)^{\alpha_{2}}\left(\varepsilon \varphi_{1}\right)^{\beta_{2}} \\
& \leq \mu_{2} b_{2}(x) u^{\alpha_{2}}\left(\varepsilon \varphi_{1}\right)^{\beta_{2}} .
\end{aligned}\right.
$$

and

$$
\left\{\begin{aligned}
-\Delta_{p}\left(K e_{1}\right)=K^{p-1} & \geq \mu_{1} C_{1} K^{\alpha_{1}+\beta_{1}}\left\|e_{1}\right\|_{\infty}^{\alpha_{1}+\beta_{1}} \\
& \geq \mu_{1} C_{1}\left(K e_{1}\right)^{\alpha_{1}}\left(K e_{1}\right)^{\beta_{1}} \\
& \geq \mu_{1} b_{1}(x)\left(K e_{1}\right)^{\alpha_{1}} v^{\beta_{1}} . \\
-\Delta_{p}\left(K e_{1}\right)=K^{p-1} & \geq \mu_{2} C_{2} K^{\alpha_{2}+\beta_{2}}\left\|e_{1}\right\|_{\infty}^{\alpha_{2}+\beta_{2}} \\
& \geq \mu_{2} C_{2}\left(K e_{1}\right)^{\alpha_{2}}\left(K e_{1}\right)^{\beta_{2}} \\
& \geq \mu_{2} b_{2}(x) u^{\alpha_{2}}\left(K e_{1}\right)^{\beta_{2}} .
\end{aligned}\right.
$$

The problem $\left(P_{1}\right)$ has a weak positive solution in the set $\left[\varepsilon \varphi_{1}, K e_{1}\right] \times\left[\varepsilon \varphi_{1}, K e_{1}\right]$. The proof is complete.

\section{References}

1. Ala S, Afrouzi GA, Niknam A. Existence of positive weak solution for $(p-q)$ Laplacian nonlinear systems. Proc Indian Acad Sci. 2015; 125: 537-44. Available from: https://www.ias.ac.in/article/ fulltext/pmsc/125/04/0537-0544.

2. Chu KD, Hai DD, Shivaji R. Positive solutions for a class of non-cooperative pq-Laplacian systems with singularities. Appl Mathematics Lett. 85(2018): 103-9. doi: 10.1016/j.aml.2018.05.024.

3. Giacomoni J, Hernandez J, Sauvy P. Quasilinear and singular elliptic systems, Adv Nonl Anal. 2013; 2: 1-41. doi: 10.1515/anona-2012-0019.

4. Haghaieghi S, Afrouzi GA, Sub-super solution for $(p-q)$ Laplacian systems. Bound. Value Probl. 2011; 2011(52). doi: 10.1186/1687-2770-2011-52.

5. Canada A, Drabek P, Gamez JL. Existence of positive solution for some problems with Nonlinear diffusion. Trans The Am Math Soc. 1997; 349(10): 4231-49. October 1997, Available from: https:/www.ams.org/journals/tran/1997-349-10/S0002-9947-97-01947-8/S0002-9947-97-019478.pdf. 
6. Dos Santos G, Figueiredo G, Tavares L. Sub-super solution method for nonlocal systems involving the $p$ (x)-Laplacian operator. Electron J Differential Equations. 2020(25): 1-19. Available from: https://ejde.math.txstate.edu/Volumes/2020/25/santos.pdf.

7. Chen $\mathrm{CH}$. On positive weak solutions for a class of quasilinear elliptic systems, Nonlinear Anal. 62

The sub-super solution method (2005): 751-56. doi: 10.1016/j.na.2005.04.007.

\section{Corresponding author}

Mohammed Moussa can be contacted at: mohammed.moussa@uit.ac.ma

For instructions on how to order reprints of this article, please visit our website:

www.emeraldgrouppublishing.com/licensing/reprints.htm

Or contact us for further details: permissions@emeraldinsight.com 